\title{
Aumento del riesgo de consultas cardiovasculares por contaminación atmosférica por partículas. Estudio en la ciudad de Santiago.
}

\author{
Oscar Román A2, María José Prieto C1, Pedro Mancilla F1, Pedro Astudillo 01, Claudio

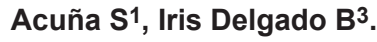 \\ 1 Unidad de Salud Respiratoria .Ministerio de Salud \\ 2 Servicio Cardiovascular. Hospital San Borja Arriarán. \\ ${ }^{3}$ Facultad de Medicina; Clínica Alemana. Universidad del Desarrollo.
}

\section{Resumen}

Introducción: En la literatura se reconoce que la contaminación del aire por partículas en diversas ciudades determina un exceso del riesgo de enfermedades cardiovasculares. Santiago es una de las urbes con mayor polución de partículas MP 10 y MP 2,5 en el mundo.

Objetivo: Estudiar la asociación entre la concentración diaria de partículas y la morbilidad cardiovascular en los 6 Servicios de Urgencia de la ciudad.

Métodos: Se consideraron el número total de consultas cardiovasculares en el año 2007, el Infarto miocárdico, accidente cerebro-vascular y crisis hipertensiva. La concentración de partículas se obtuvo de los registros de 7 centros de la red de Monitoreo de la Calidad del Aire de la Región Metropolitana (MACAM) y las variables temperatura y humedad, del Instituto de Meteorología de Chile. Estadísticamente se implementó un modelo de regresión múltiple GAM.

Resultados: Se observó que para todo el año 2007 las concentraciones de partículas ambientales MP 10 estuvieron altas, sobre la norma recomendada por la OMS. Ello se asoció a un incrementó del 10\% del riesgo de consultas cardiovasculares durante todo el año 2007. Para las partículas MP 2,5 el riesgo aumentó en un $17 \%$. En los meses invernales Junio y Julio, el riesgo aumentó en $15 \%$ y $28 \%$ respectivamente. Todas las cifras resultaron altamente significativas ( $p$ 0.001). Para la crisis hipertensiva, los aumentos del riesgo fueron $4 y$ $13 \%$, también significativos.

Conclusión: Se concluye que la contaminación por partículas se asocia significativamente a un mayor riesgo de consultas por enfermedades cardiovasculares en la ciudad de Santiago y que el riesgo es mayor en presencia de partículas MP 2,5.

\section{Increased risk of consultation for cardiovascular problems related to particle atmospheric pollution. A study in Santiago, Chile.}

Background: Atmospheric contamination due to particles is recognized as a factor related to an increased risk of cardiovascular disease. Santiago has one of the greatest concentrations of MP 10 and MP 2.5 particles in the world.

Aim: to determine the association of daily changes in particle concentration and cardiovascular morbidity in 6 emergency consultation centers in Santiago.

Correspondencia: Oscar Román A.

Huelen 154 Dto 2. - Providencia - Santiago, Chile

Fax 5567576

Correo Electrónico:Oscarromanalemany @hotmail.com 
O.Román, M. J. Prieto, P. Mancilla, P. Astudillo, C. Acuña, I. Delgado.

Methods: Total number of consults, myocardial infarction, stroke and hypertensive crisis were included as endpoints. Concentration of particles was obtained from registries routinely made in 7 centers of a city based environmental agency ("Monitoring quality of air in the Metropolitan Region", MACAM). Air temperature and humidity were obtained from the Chilean Meteorology Institute. Data were analyzed using a multiple regression model (GAM).

Results: Throughout 2007, concentration of MP 10 was above the norm recommended by WHO. This was associated to a $10 \%$ increase in CV related consultations. In relation to MP 2.5 the risk was increased $17 \%$. The CV risk peaked at $15 \%$ and $28 \%$ in June and July, respectively. All relations were highly significant $(p<0.001)$ including the 4 and $13 \%$ increase in hypertension emergencies.

Conclusion: Increased particle concentration in the air is significantly associated to a higher risk of cardiovascular consultation in Santiago. The risk is higher in relation to increased MP 2.5 particle concentration.

Key words: air polution, cardiovascular morbidity

Recibido el 21 de abril de 2009. Aceptado el 10 de junio de 2009

\section{Introducción}

Desde la década de 1980 se conoce que la contaminación atmosférica por partículas determina mayor mortalidad y morbilidad por afecciones respiratorias en diversas ciudades del mundo 1,2 , y que esa asociación epidemiológica también se presenta en la ciudad de Santiago 3,4. Sin embargo, el reconocimiento que la contaminación también produce un efecto sobre las enfermedades cardiovasculares ha sido posterior. Ostro ${ }^{5}$ y Sanhueza 6 , a fines de los 90 , en la cuenca de la ciudad de Santiago comprobaron que las altas concentraciones diarias de partículas grandes (MP 10), se asociaban no sólo a incrementos de la morbimortalidad respiratoria sino también cardiovascular. En la literatura internacional se ha comunicado una mayor frecuencia de hospitalizaciones y muertes de origen $\mathrm{C}-\mathrm{V}$ durante los períodos de elevada concentración de partículas contaminantes, como ocurre en la estación invernal $7,8,9$.

Respecto a la fisiopatología de la alteración C-V, se han postulado como mecanismos posibles, por un lado, la inflamación sistémica endotelial producida por el paso a través de los alvéolos pulmonares de I as partículas dañinas, provocando inflamación y estimulación de factores protrombóticos, como ha postulado Jalil en Santiago en pacientes coronarios y por otro, un desequilibrio del sistema autonómico10,11,12. Santiago es una ciudad con alta contaminación por

partículas grandes (MP10) y pequeñas (MP 2,5), que alcanzan concentraciones máximas y cuyo promedio diario triplica los estándares de la OMS, que son de $50 \mathrm{ug} / \mathrm{m} 3$ para MP 10 y de $20 \mathrm{ug} / \mathrm{m} 3$ para MP 2,513. Presenta, además, marcadas diferencias de temperatura entre las diversas estaciones del año5,14. Con estos antecedentes nos ha parecido importante estudiar la relación entre la concentración diaria de las partículas contaminantes y la morbilidad aguda de las enfermedades cardiovasculares en la ciudad de Santiago en el año 2007.

\section{Metodología}

Establecimos como hipótesis que las concentraciones de partículas atmosféricas MP 10 y MP 2,5 influyen en el número de consultas diarias de enfermedades cardiovasculares en la ciudad de Santiago. El estudio se realizó durante el año 2007. La información sobre las variables de contaminación se obtuvo del registro diario de la concentración de ambos tamaños de partículas en los 7 centros de la Red de Monitoreo de Calidad del Aire en la región Metropolitana (MACAM), estableciendo como variables de contaminación los valores máximos de ambas partículas, MP10 y MP 2,5, expresadas en ug/m3. Además se registraron las temperaturas máxima y 
mínima y el valor máximo de la humedad relativa del aire, obtenidos del Instituto de Meteorología de Chile.

El número diario de consultas (morbilidad) por causas cardiovasculares se obtuvo de los registros de los 6 Servicios de Urgencia hospitalarios de la ciudad de Santiago, los que fueron consolidados diariamente por el Departamento de Estadística e Información de Salud (DEIS) del Ministerio de Salud. El total de consultas cardiovasculares en el año fue de 34.864, cuyo desglose por enfermedades más prevalentes se anota en la Tabla 1. Los diagnósticos fueron esta- blecidos por los médicos de los respectivos Servicios de Urgencia, de acuerdo a los criterios habituales.

Para el análisis estadístico se implementó un modelo de regresión múltiple aditivo (GAM) y ajustado a la distribución de los datos (Poisson). El modelo se aplicó para cada uno de los diagnósticos C-V (variables dependientes), utilizando como variables independientes MP 10 y MP2,5, controlando por las variables tem-peratura del día y temporalidad (día observado) ${ }^{14}$. Los cálculos fueron aplicados para el año completo y para los meses de junio y julio, en los cuales se observa una mayor concentración de partículas.

Tabla 1: Consultas cardiovasculares registradas en 6 servicios de urgencia de santiago año 2007

\begin{tabular}{|c|c|}
\hline Infarto agudo miocárdico & 2.233 \\
\hline Accidente cerebro-vascular & 6.286 \\
\hline Crisis hipertensiva & 19.927 \\
\hline Arritmias & 3.427 \\
\hline Otros & 8.081 \\
\hline Total consultas C-V.......... & 34.864 \\
\hline Total meses de Junio y Julio & 5.982 \\
\hline
\end{tabular}

\section{Resultados}

En la Tabla 2 se muestran las variables estudiadas. La concentración media de ambos tipos de partículas en el año estuvo sobre la norma internacional, que es de 50 y $20 \mathrm{ug} / \mathrm{m} 3$, respectivamente. Se observó que los valores máximos para el año superan 5 veces a la norma. El rango de temperaturas máxima y mínima fueron los habituales de la ciudad de Santiago, como también la humedad relativa.

Tabla 2: Variables de contaminación del aire Santiago, 2007

\begin{tabular}{ll}
\hline VARIABLE & MEDICION \\
\hline MP 10 Concentración media anual.......... & $69 \mathrm{ug} / \mathrm{m3}$ \\
MP 2,5 Concentración media anual.......... & $32 \mathrm{ug} / \mathrm{m3}$ \\
Temperatura mínima media & $7,7(-3 / 16) \mathrm{C}^{\circ}$ \\
Temperatura máxima media & $22,2(7 / 34) \mathrm{C}^{\circ}$ \\
Humedad & $66,6(26 / 99)$ \\
\hline
\end{tabular}

En cuanto a las consulta C-V, la frecuencia de Infarto miocárdico registrada por el DEIS fue baja, probablemente debido a que fueron informados sólo los infartos completamente definidos en el momento de la consulta, en base a la clínica, el ECG y las enzimas, de modo que no se informaron los síndromes isquémicos en comienzo.

La Tabla 3 muestra el riesgo relativo del total de eventos cardiovasculares y de las crisis hipertensivas en relación a las concentraciones de MP 10 y MP 2,5, separadamente para todo el año y para los meses de Junio y Julio. 
O.Román, M. J. Prieto, P. Mancilla, P. Astudillo, C. Acuña, I. Delgado.

Tabla 3: Aumento del riesgo de enfermedades cardiovasculares por contaminación atmosférica. santiago, 2007

\begin{tabular}{|c|c|c|c|c|}
\hline \multirow[t]{2}{*}{ Variable C-V } & \multicolumn{2}{|c|}{ Total 2007} & \multicolumn{2}{|c|}{ Junio-Julio 2007} \\
\hline & PM 10 & PM 2,5 & PM 10 & PM 2,5 \\
\hline Total Enfermedades C-V & $10 \%$ * & $17 \%$ * & $15 \%$ * & $28 \%$ * \\
\hline Crisis Hipertensiva & $4 \%$ * & - & $7 \%$ * & $13 \% \circ$ \\
\hline${ }^{*} p<0.001$ & & & & \\
\hline
\end{tabular}

Se observa que para el total de eventos cardiovasculares el aumento del riesgo fue del $10 \%$ en relación a la concentración de MP 10 y del $17 \%$ para MP 2,5 , ambos incrementos significativos $(p<0.001)$. Para los meses de Junio y Julio, el aumento del riesgo asociado a MP 10 fue del $15 \%$ y del $28 \%$ para MP 2,5, ambos porcentajes significativamente mayores que los correspondientes a todo el año $(p<0.001)$.

Respecto a la crisis hipertensiva, el aumento del riesgo asociado a MP 10 fue del $4 \%(p<0.001)$ y no significativo para MP 2,5. Sin embargo, en los meses invernales el aumento de riesgo fue significativamente mayor para ambas partículas, correspondiendo un $7 \%$ a MP $10(p<0.001)$ y un $13 \%$ a MP 2,5 $(p<0.03)$.

\section{Discusión y Comentario}

El análisis realizado en el año 2007 para la ciudad de Santiago, demuestra una asociación estadísticamente significativa entre el número total de consultas por eventos $\mathrm{C}-\mathrm{V}$ y el valor máximo diario de partículas MP10 y MP 2,5. Esta asociación también se observó para el número de consultas por crisis hipertensiva. Dado el menor número de consultas registradas para el infarto miocárdico y el accidente cerebrovascular, el análisis no permitió conclusiones estadísticas para esas patologías.

Una limitación del estudio es que los registros de las consultas en $S$ de $U$ de la RM por causa específica CV se inician en año 2007, por lo que no se cuenta con datos previos para hacer comparaciones, sin embargo estos continúan realizándose lo que permitirá a futuro una mirada más precisa. Otra posible limitación es la certidumbre del diagnóstico que es realizado por médicos no especialistas, pero que tienen amplia experiencia en el diagnóstico y manejo de estas frecuentes morbilidades.

En la literatura existe abundante información de la asociación entre contaminación del aire por partículas y eventos cardiovasculares, pero la gran mayoría de ella se refiere a cifras de mortalidad. Los datos comunicados respecto a morbilidad son escasos. Entre ellos, el estudio de 6 ciudades en EE. UU reveló que la ciudad más contaminada presentó un $26 \%$ de aumento del riesgo de enfermedades $\mathrm{C}-\mathrm{V}$ en relación a las menos contaminadas ${ }^{15}$.

En una ciudad de Brasil, el incremento del riesgo C-V en exposición aguda a MP 10 fue del $4 \%(p<0,05)^{16}$. En un estudio en mujeres en la ciudad de Seattle, EE.UU, el aumento de riesgo de eventos C-V fue del $24 \%$ por cada aumento de $10 \mathrm{ug} / \mathrm{m} 3$ de MP2,517. En la mayoría de las comunicaciones revisadas el aumento de riesgo se calcula en relación a cada incremento de $10 \mathrm{ug} / \mathrm{m} 3$ en la concentración de partículas. Con ese criterio, el incrementos de riesgo de muerte para el total de enfermedades C-V fluctúa entre un $0,4 \%$ y un $1 \%$, y específicamente para la enfermedad coronaria, el accidente cerebrovascular y las arritmias, entre un $4,5 \%$ y un $18 \% 17,18,19,20$

Es preciso destacar que la mayoría de los estudios se refieren a las partículas grandes o MP 10, pero aquellos que registraron las partículas pequeñas, $O$ MP 2,5, demostraron un significativo riesgo mayor8.

Con respecto a la ciudad de Santiago, nuestros datos confirman lo observado por Ostro y Sanhueza, en la década de los 90 , que se referían a mortalidad por enfermedades cardiovasculares 5,6 . Nuestros resultados se basan en registro de morbilidad $\mathrm{C}-\mathrm{V}$, y por tanto en un espectro mayor de población, lo que determina una alerta para enfrentar la acción patológica de la contaminación del aire.

Además, la asociación registrada entre daño C-V 
y contaminación atmosférica por partículas, sugiere la aparición de un nuevo factor de riesgo de las enfermedades $\mathrm{C}-\mathrm{V}$, por lo que es importante realizar nuevos estudios al respecto.

Pero desde el punto de vista de la salud pública, aparece imperativo realizar un esfuerzo multifactorial para reducir los niveles de contaminación del aire, no sólo en Santiago, sino también en otras ciudades del país.

Si bien, no hemos podido demostrar significativamente una asociación entre contaminación y morbilidad por enfermedad coronaria, la literatura registra una acción deletérea mayor en los pacientes con ateroesclerosis coronaria, demostrada por coronariografía, en relación a quienes no la presen$\tan 19,20$. Entre nosotros, Jalil y col11, en pacientes coronarios expuestos a contaminación por MP 2,5 en la ciudad de Santiago, observaron una serie de cambios fisiopatológicos que pueden llevar a obstrucción coronaria, consistentes en stress oxidativo, inflamación vascular y fenómenos protombóticos 10,11,20,21,22.

Desde el punto de vista de la Salud Pública, las concentraciones de ambos tipos de partículas, MP 10 y MP 2,5, observadas en el año 2007, superan las normas internacionales. Ello pone una nota de alerta ambiental, pues la norma vigente en Chile es de $150 \mathrm{ug} / \mathrm{m} 3$ para MP 10, tres veces superior a la internacional, la que seguirá vigente hasta 2010 en el plan de descontaminación de Santiago ${ }^{14}$.

En conclusión, esta comunicación confirma estudios nacionales anteriores en la ciudad de Santiago, coincide con numerosas observaciones internacionales y pone una nota de preocupación respecto a las acciones a ejecutar para mejorar la condición ambiental de la ciudad capital.

\section{Referencias}

1. MAZUMDAR S, SCHIMMEL H, HIGGINS I. Relationship of daily mortality to air pollution: an analysis of 14 London winters, 1958.59/ 1971-72. Arch Environ Health 1982; 38: 213-20.

2. OSTRO BD, Air pollution and acute respiratory morbidity: an observational study of multiple pollutants. Environ Res 1989; 50: 238-47.

3. ARANDA C, ASTUDILLO P. Correlación entre contaminación por MP 10 y mortalidad por neumonia en niños de Santiago. Rev Ch Enf Respir 1991; 7: S33.

4. PRIETO MJ, MANCILLA P, ASTUDILLO P, ROMAN O. Exceso de morbilidad respiratoria en niños y adultos mayores en una comuna de Santiago con alta contaminación atmosférica. Rev Med Chile 2006; 135: 221-28

5. OSTRO B, SANCHEZ JM, ARANDA C, ESKELAND GS. Air pollution and mortality: results from a study of Santiago, Chile. J Expos Anal Environ Epidemiol 1996; 6: 97-114.

6. SANHUEZA P, VARGAS C, JIMENEZ J. Mortalidad diaria en Santiago y su relación con la contaminación del aire. Rev Med Chile 1999; 127: 235-42.

7. PETERS A, DOCKERY DW, MULLER JE. MITTLEMAN MA. Increase particulate air pollution and the triggering of myocardial infarction. Circulation 2001, 103: 2810-15.

8. SAMET JM, DOMINICI F, CURRIERO I, ZEGER SL. Fine particulate air pollution and mortality in 20 US cities 1987-1994. N Engl J Med 2000; 343: 1742-9.

9. ZANOBETTI A, SCHWARTZ J, DOCKERY DW. Airborne particles are a risk factor for hospital admissions for heart and lung disease. Environ Health Perspect 2000; 108; 1071.77.
10. POPE CA 3erd, BURNET RT, THURSTON GD, THUN MJ, CALLE E, KREWSKY D. et al. Cardiovascular mortality and long term exposure to particulate air pollution: epidemiological evidence of general pathophysiological pathways of disease. Circulation 2004; 109: 71-7.

11. JALIL J, DUMASA E, CIFUENTES L, OCARANZA MP, CHAMORRO G, PADILLA I, ET AL. Desbalance autonómico y procoagulación debido a contaminación atmosférica en Santiago: un estudio prospectivo. Rev Chil Cardiol 2003; 22: 223-32.

12. America heart association professionals from the expert panel on population and prevention science of the air pollution and cardiovascular disease. A statement for health care. Circulation 2004; 109: 2655-71.

13. WHO. Air quality guidelines. Global update 2005. www europubwho.int

14. DIARIO OFICIAL DTO 58. Ministerio Secretaria General de la Presidencia. Reformula y actualiza0 Plan de Prevención y Descontaminación atmosférica Para la Región Metropolitana. Enero 2004.

15. DOCKERY DW, POPE CA 3RD, XU X, SPENGLER JD, WARE JH, FAY ME, et al. An association between air pollution and mortality in six U.S. cities. $\mathrm{N}$ Engl $\mathrm{J}$ Med 1993, 329: 1753-59.

16. BRAGA AL, PEREIRA LA, PROCOPIO M, ANDRE PA, SALDIVIA PH. Association between air pollution and $r$ espiratory and cardiovascular diseases in Itabira, Minas Gerais State, Brazil. Cad Saude Publica. 2007; 23:S570-8. 
O.Román, M. J. Prieto, P. Mancilla, P. Astudillo, C. Acuña, I. Delgado.

17. MILLER KA, SISKOVICK DS, SHEPPARD L, SULLIVAN JH, ANDERSON GL KAUFMAN JD. Long term exposure to air pollution and incidence of cardiovascular events in women. N Engl J Med 2007; 356: 447-58.

18. KARSOUYANNIO K, TOULOUMI G, SPIX C. Short term effects of ambient sulphur dioxide and particulate matter on mortality in 12 European cities: results from time series data from the APHEA project: Air pollution and Health: a European approach. Brit Med J 1997; 314:1658-63.

19. NAWROT TS, NEMMAR A, NEMERY B. Air pollution: to the heart of the matter. Europ Heart J 2006; 27: 2269-71.
20. POPE CA 3erd, MUHLESTEIN JB, MAY HT, RENLUND DG, ANDERSON J L, HORNE BD. Ischemic heart disease events triggered by short term exposure to fine particulate air pollution. Circulation 2006; 114: 2443-48.

21. BROOK RD, FRANKLIN B, CASCIO W, HONG Y, HOWARD DG, LIPSETT $M$. et al. Air pollution and cardiovascular disease. A statement for health care professionals from the expert panel on population and prevention science of the American Heart Association Circulation 2004; 109: 2655-71.

22. KELLY FJ. Oxidative stress: its role in air pollution and adverse heath effects. Occup Environ Med 2003; 60: 612-16. 